\title{
A Public Health Messaging Campaign to Reduce Caloric Intake: Feedback From Expert Stakeholders
}

\author{
Sarah Gonzalez-Nahm, PhD, MPH, RD*; Anam M. Bhatti, MPH*; \\ Meghan L. Ames, MSPH, RD; Daniel Zaltz, MPH; Sara E. Benjamin-Neelon, PhD, JD, RD
}

\begin{abstract}
Objective: To obtain expert feedback on a public health messaging campaign to reduce caloric intake in US adults.

Design and Setting: In 2018, researchers conducted semistructured telephone interviews with US-based experts in obesity prevention, mental health, and health communications.

Participants: The research team invited 100 experts to participate using purposive and snowball sampling techniques. Of those invited, 60 completed interviews, among which 37 (62\%) were obesity prevention experts, 12 (20\%) were mental health experts, and 11 (18\%) were health communications experts.

Main Outcome Measure: Expert feedback regarding a public health messaging campaign to reduce caloric intake.

Analysis: Two researchers reviewed and coded all transcripts. The team identified major themes and summarized findings.

Results: Most experts identified barriers to effective calorie reduction including social and environmental factors, lack of actionable strategies, and confusion regarding healthy eating messages. Expert suggestions for effective messaging included addressing eating patterns, emphasizing nutrient density, and dissemination through multiple channels and trusted sources. In general, mental health experts more frequently voiced concerns regarding eating disorders, and communications experts raised issues regarding the dissemination of campaigns.

Conclusions and Implications: Professionals should identify and address barriers to delivering a calorie reduction campaign before implementation, using strategies that enhance delivery to ensure an effective campaign.
\end{abstract}

Key Words: health communication campaign, calorie reduction, nutrition, formative research $(J$ Nutr Educ Behav. 2020; 52:595-606.)

Accepted February 26, 2020. Published online April 13, 2020.

\section{INTRODUCTION}

Average portion sizes in the US have increased dramatically in recent decades and have contributed to the rising prevalence of obesity among adults. Several studies have demonstrated that large portion sizes have a powerful effect on calories consumed. $^{1-4}$ This effect is more pronounced with energy-dense food and remains consistent regardless of demographics, such as age, sex, race, and body mass index. ${ }^{5}$ The portion size effect could be explained, in part, by the amount of food people deem socially acceptable to eat. ${ }^{6-8}$ Changing people's perceptions about socially acceptable portion sizes may be 1 important way to help decrease consumption.

Public health messaging campaigns designed to reduce calorie intake and portion size have the potential to

Department of Health, Behavior and Society, Johns Hopkins Bloomberg School of Public Health, Baltimore, MD

^These authors contributed equally to this work.

Conflict of Interest Disclosure: The authors have not stated any conflicts of interest.

Address for correspondence: Sarah Gonzalez-Nahm, PhD, MPH, RD, Department of Health, Behavior and Society, Johns Hopkins University, 615 North Wolfe St, Baltimore, MD; E-mail: sneelon@jhu.edu

(C) 2020 The Authors. Published by Elsevier Inc. on behalf of Society for Nutrition Education and Behavior. This is an open access article under the CC BY-NC-ND license. (http://creativecommons.org/licenses/by-nc-nd/4.0/)

affect health behaviors on a population level by changing perceptions about the appropriate amount of food to consume. $^{9-11}$ Formative evaluations provide data to improve campaign acceptability, answer questions about the target audience, and inform the best method to disseminate the message. ${ }^{12,13}$ However, information on formative evaluation of nutrition campaigns is generally limited. A systematic review of adult-targeted obesity campaigns within high-income countries found information on formative evaluation for only 4 of 13 campaigns. ${ }^{13}$ Formative evaluation methods primarily focus on message testing with focus groups or select experts through an advisory group. ${ }^{12,13}$ For example, the Pouring on the Pounds campaign to reduce sugary drink consumption among San Francisco, CA residents engaged 7 key informants from the Department of Health and local nonprofit organizations. ${ }^{14}$ 
Similarly, The Choose Less Weigh Less Portion Size Matters campaign launched by the Los Angeles County Department of Public Health included key informant interviews in the formative evaluation methods, but the findings were not available to the public. ${ }^{10}$ Results on formative evaluations are rarely published or shared with the public, and very little information is provided on how formative evaluation findings are incorporated into campaign design. ${ }^{13}$

The use of expert feedback in the development of public health messaging campaigns could improve campaign outcomes. Expert feedback may also help reduce unintended consequences, such as stigma, and improve receptivity of messages by different groups. One way to craft an appropriate public health message related to reducing caloric intake is to engage a variety of health experts, within nutrition, mental health, and health communications, to help ensure that messages are widely accepted by diverse audiences. ${ }^{15}$ The purpose of this study was to obtain expert feedback on potential barriers and strategies to delivering an effective public health messaging campaign to reduce caloric intake in the US. Secondarily, the research team assessed expert beliefs about important cultural and contextual factors that may affect caloric intake and expert feedback about how a calorie reduction messaging campaign should be designed and disseminated to a broad adult audience.

\section{METHODS}

\section{Study Design}

This study was part of a multiphase formative research project to explore the attitudes, knowledge, and beliefs regarding a specific public health messaging campaign to reduce caloric intake. Between August and December, 2018, researchers conducted semistructured telephone interviews with experts in 3 domains: (1) obesity prevention, (2) mental health, and (3) health communications. The research team defined an expert as someone who had a leadership role in 1 of the 3 domains and experience using, developing, or informing health promotion messages within the US. The team interviewed experts in these domains because their experience and collective expertise provided a unique and comprehensive perspective on calorie reduction messages. The research team included 2 doctoraltrained researchers, 2 staff members with master's degrees in public health, and 1 public health graduate student. All team members had some degree of qualitative training. The study protocol was considered exempt by the Johns Hopkins University Institutional Review Board.

\section{Participants and Recruitment}

Members of the research team identified 100 experts to participate in the study using purposive and snowball sampling techniques. The research team developed an initial list of 62 experts from authors of published studies in the literature and through the research team's professional networks relevant to this study. Members of the research team sent requests to experts via e-mail, including an attached letter detailing the purpose of the study. The team asked experts to respond if they were interested in participating. From these initial contacts, $40(64.5 \%)$ agreed to participate, and $22(35.5 \%)$ declined. To ensure sampling beyond the initially identified experts, the research team implemented snowball sampling, in which participating and declining experts were asked to refer qualified colleagues in 1 of the 3 domains. The team identified an additional 38 potential respondents (16 obesity prevention experts, 8 mental health experts, and 14 communications experts) through snowball sampling. Of these, 20 (52.6\%) experts agreed to participate in the study ( 8 obesity prevention experts, 3 mental health experts, and 9 communications experts).

Of the 100 total identified experts, 33 did not respond to the e-mail invitation, 7 declined, and 60 agreed to participate in the study. Among total nonresponders $(n=33), 16(48.5 \%)$ were obesity prevention experts, 7 $(21.2 \%)$ were mental health experts, and $10(30.3 \%)$ were communications experts. Among those who declined participation, 5 (71.4\%) were obesity prevention experts, 1 $(14.3 \%)$ was a mental health expert, and $1(14.3 \%)$ was a communications expert. The research team did not collect data on reasons for declining participation. Researchers did not provide experts with an incentive for participation. Experts provided verbal consent to complete the interview and participate in the study.

\section{Interview Process}

The research team developed a 17question semistructured interview guide to allow for flexibility in responses, while gathering pertinent information from respondents during a single interaction. The research team did not pilot-test interview questions but did use the results from a previous quantitative survey of 3,903 US adults on portion control messaging (unpublished survey results, fall 2018), preliminary results from 17 focus groups of 113 US adults testing a calorie reduction message (unpublished focus groups, summer 2018), and a literature review of existing public health messaging campaigns to reduce caloric intake to inform the survey. ${ }^{9-11,15-18}$ Interview respondents were asked to provide their reactions and feedback on a calorie reduction campaign message encouraging individuals to "eat less." Moreover, the interview guide covered factors that may influence portion control and dietary behaviors of adults, barriers in developing a portion control messaging campaign, strategies to overcome these barriers, and periodicity of health behaviors. Three members of the research team with qualitative research experience reviewed and edited the interview script. The research team used the same questions across all interviews; however, a flexible conversation format allowed the interviewer to delve deeper into new issues brought up by experts. Each expert completed a semistructured telephone interview that lasted approximately 30 minutes. The interviewer did not conduct respondent validation; however, the semistructured interview format allowed the interviewer to ask followup questions when a response was not clear. One member of the research 
team conducted all interviews and determined when redundancy in expert response occurred. Once the team reached saturation, they conducted additional interviews within each domain to ensure consistent responses and exhaustion of themes. The consistency of themes throughout each domain suggested the reliability of the data.

\section{Data Coding and Analysis}

An external transcription company professionally transcribed each interview. One research team member (A. B.) then reviewed the transcripts for accuracy and imported them into Atlas.ti 8.3.16 (Scientific Software Development GmbH, Berlin, Germany, 2018). The research team employed a general inductive approach to coding transcripts based on broad themes, as determined by the study aims. Two research team members (A.M.B. and M.L.A.) and a graduate student were responsible for independently coding the data. One research team member (A.M.B.) coded all the interview transcripts, whereas the other 2 each coded half. Two research team members (A.M.B. and M.L.A.) independently reviewed 3 random transcripts and highlighted key concepts to assign the initial codes. The research team met to reach consensus by discussing the proposed codes, comparing findings, and revising the code book. The team reached consensus on any code discrepancies to ensure inter-rater reliability. The research team assigned multiple codes to statements that fit into more than 1 thematic area. Once all documents were coded, the research team met regularly to discuss themes emerging from the data. Team members discussed how the themes might be conceptually linked and combined in a logical way. In most instances, researchers agreed on the themes. However, when there were disagreements, the team discussed each researcher's thought process and perspective. A member of the research team not involved in the coding attended team meetings to provide an additional perspective and ensure the emerging themes were supported by the data. Researchers kept a log of quotes that supported emerging themes and did not identify any quotes that contradicted or invalidated any of the themes presented. The research team identified several themes, which they categorized as either barriers or strategies to developing an effective public health messaging campaign to reduce caloric intake. The research team also identified expert guidance on how a public health messaging campaign should be disseminated.

\section{RESULTS}

Among experts, 37 (62\%) were obesity prevention professionals, 12 (20\%) were mental health professionals, and 11 (18\%) were health communications professionals (Table). Forty-five experts had a doctoral-level degree, 11 had a master's degree, and 4 had a bachelor's degree. The team interviewed 20 (33.3\%) experts recruited through snowball sampling. Among these, 9 (45\%) experts were communications professionals, $8(40 \%)$ were obesity prevention experts, and 3 (15\%) were communications experts. Not all experts identified the same barriers, strategies, or suggestions for dissemination. However, no experts provided responses that contradicted the themes presented. In general, mental health experts more frequently brought up issues related to eating disorders when discussing calorie reduction campaigns. Alternatively, communications experts brought up more issues related to the dissemination of communications campaigns.

\section{Barriers to Implementation of Calorie Reduction Campaign}

Many experts identified 3 barriers that could impede the success of a public health messaging campaign to reduce caloric intake: (1) the complex social, environmental, and cultural factors that influence intake; (2) lack of actionable strategies to effectively reduce calories; and (3) the existence of too many, often conflicting, messages.

Complex social and environmental factors that influence intake. Experts in this study noted that the social and structural factors that influence dietary behavior can be important barriers to creating a successful calorie reduction campaign. In particular, experts believed it was important to consider the unique needs of communities struggling with food insecurity and competing priorities and individuals with eating disorders. One obesity prevention expert (\# 41) summarized:

\section{[Among] many of the low-income populations we work with, there's a lot of bigger priorities going on in their lives than nutrition. Some things very immediate, and much}

\section{Table. Characteristics of Experts Interviewed $(n=60)$}

\section{Characteristic}

Expert domains

Communications

Mental health/eating disorder prevention

Nutrition and obesity prevention

Employment type

Academia and research

Advertising Agency

Advocacy Organization

Community-based organization

Government

Food and hospitality services

Hospital or health center

Non-for-profit

Academic credentials

Doctoral degree

Master's degree

Bachelor's degree 
more concerning, such as where we're going to live, and how we're going to make it, financially, through the month, and getting enough food. And food is, while still expensive, it's a relatively cheap way to treat yourself. So, for many folks, that's not something they want to change, because so many other parts of their everyday are challenged.

One mental health expert (\# 44) further emphasized the influence of food insecurity on dietary choices related to portion sizes,

\begin{abstract}
...one large barrier, particularly where I work, is food insecurity in general. That's, when folks do get food, the, I don't know if culture is the right word, but it's "let's eat what's there because this is here now and we don't know when we're gonna eat again." So we see that with proportion sizing but also the types of food... I think, thinking about portion sizing or eating less or eating more is difficult when you're really just trying to survive.
\end{abstract}

Another mental health expert (\# 46) also brought up the role of competing priorities in dietary choices,

\section{... some ladies we work with in our obesity related programming... they know they're overweight. That's why they enrolled in the program. They're trying to do something but it's hard because they have little kids and they have a lot of other competing things.}

Experts cautioned that a calorie reduction campaign that did not account for these factors could isolate vulnerable groups and even have unintended negative consequences, particularly for those struggling with eating disorders or food insecurity.

A communications expert (\#54) cautioned against using a generic calorie reduction message in food insecure populations,

And so it's a struggle because when you're talking about really lowincome communities... If you're talking about people who are literally starving saying eat less is not the right message. So we really need to think about that from a health equity perspective of it's not that people necessarily need to eat less.

When asked about potential negative consequences of a calorie reductions message, the concerns of this mental health expert (\# 11) highlighted how the message may affect individuals with eating disorders,

Well, I think that patients with an eating disorder may use this to rationalize their behavior. They use a lot of distorted logic to rationalize what they do and things like, "Well, I'm able to control my eating, but two-thirds of the population is not, so my eating is better than theirs" even if they're vomiting or are really underweight and have osteoporosis, so this message actually could be construed as reinforcing to someone with an eating disorder.

Another mental health expert (\# 20) echoed this sentiment,

Somebody with an eating disorder or in the midst of developing an eating disorder, the message to eat less is just sort of like, I don't know, putting a banana peel on a puddle of ice and having them step on it. It's really going to reinforce messages that are not necessarily true.

Lack of actionable strategies to effectively reduce calories. Across the 3 domains, experts thought that many adults were unmotivated to reduce their caloric intake because they lacked knowledge about how to effectively reduce calories without feeling deprived. A message lacking specific, actionable strategies could lead to unintended consequences, such as the reduction of healthy food intake or extreme deprivation followed by binge eating. As 1 obesity prevention expert (\# 4) explained,

I think [a calorie reduction message] induces a sense of deprivation, even if it's not physical deprivation, it's a fear of deprivation and I think when people hear that, they think about what they can cut out of their diet, they don't think about what they can increase in their diet, even though the result may be actually lower calorie, that they're using foods that are less calorie dense. So, I think it can actually set people up for last supper eating and even possible binge eating, it's the fear of deprivation.

A mental health expert (\# 5) emphasized this thought with the following quote:

I do think there is a case for simplicity, so I think that is good, but, yeah, I think it needs kind of more sort of behaviorally based and behaviorally sound kind of specific things people could try. I do think people find different things useful...

With respect to eating disorders, another mental health expert (\# 11) added:

Well, the problem with that of course...is that we also have a portion of the population that eats too little because they have anorexia, so I think "Eat less" is not clear enough.

Experts also believed that individuals were aware of the relationship between calorie consumption and obesity but felt unsure about the number of calories they should consume or how to reduce them in a healthful way. A communications expert (\# 32) elaborated,

I wouldn't throw calories out the window completely, I just think people need a way of understanding what the numbers mean with calories. Like what does 1,000 calories mean? I just think this is an area where the health literacy I think is really poor.

A mental health expert (\# 25) emphasized this with the following quote:

I think visual ways of communicating messages about portion size, I think, are probably very important...because all of the research that we are aware of shows that most people are really challenged to understand numeric concepts around nutrition, and portion sizes in general are just really hard for people to grasp. 
The existence of too many, often conflicting, messages. Finally, experts thought that there were too many, often conflicting, messages already in existence. They believed messages about calories, types of food to eat or avoid, and diet restrictions came from various sources, such as the food industry, popular food culture, and health organizations. Experts noted that the oversaturation of messages can lead to confusion and apathy. One communications expert (\# 44) said,

What we hear from a lot of folks in communities is that I'm being told one thing, and then, next week, I'm being told something different. Or in some cases... maybe it's slightly different, sometimes it's the total opposite of what I was just told to do.

A mental health expert (\# 10) added:

So it's kind of - I think that it's there's so many messages out there about how to eat healthfully and how to manage your weight that it's very hard just to send one message.

An obesity prevention expert (\# 2) shared their experience with the public's confusion and potential apathy because of conflicting health messages:

So they hear messages related to, "You should not eat this. You should eat fewer of these. You should eat a little bit of this," and then we change, you know, the research changes or there's some doubt placed on it and then we say something else, and people are so confused that they choose to do nothing, and in fact...people have told us, you know, "When you get this right and tell me exactly what I need to focus on and do to reduce my calorie consumption and my sugar consumption, I'll do it, but until that time, you don't all seem to be on the same page as far as what we should be working on."

\section{Strategies for Implementation of Calorie Reduction Campaign}

Across the 3 domains, experts identified 4 strategies to create an effective calorie reduction campaign. These included the following strategies: (1) consider eating behaviors and patterns, (2) emphasize nutrient density, (3) design appealing messages, and (4) consider timing and periodicity of behaviors.

Consider eating behaviors and patterns. Experts suggested that a calorie reduction message should emphasize actionable healthy eating behaviors rather than reducing certain food. Experts identified strategies to improve eating habits, such as eating balanced meals, avoiding skipping meals, eating on a schedule, portioning meals ahead of time, and practicing mindful eating. Some of these strategies were best captured by 1 obesity prevention expert (\# 8),

I think portion size is a little different. I think that's easy for people to see and track. I think that's easy for them to use a smaller plate, use a smaller bowl, go out to dinner, divide it in half, ask the server to package-up the half to take home before you even start eating. Things like that are actionable and visually clear. Fewer calories is very tough. You don't know how many calories are in this or that. That's a hard thing, and I think people associate that with being hungry, so you're more likely to be successful I think if what you say then is to change the way you fill up, so fill up with different foods.

Experts thought that focusing on individuals' eating patterns could help address social factors that influence dietary habits. An obesity prevention expert (\# 14) suggested,

Focus more on healthy eating patterns and reflecting culturally relevant eating patterns and that kind of thing and taking a more holistic approach to health and healthy body weight and what that actually means for a given person, as opposed to just focusing on reducing caloric intake, which might not be appropriate for everybody.

Emphasize nutrient density. Experts recommended that a calorie reduction message should emphasize diet quality and nutrient density of food, rather than just caloric intake. As 1 nutrition expert (\# 23) said,

If you're just generally saying to eat less of everything, well that's not necessarily an accurate message or a sustainable message because people get hungry. We want to encourage satiety. We want to encourage eating plentiful amounts of nutrient dense foods.

A communications expert (\# 54) added:

I think that dietitians and nutritionists and public health community are pushing - have been pushing the calorie message for a long time...And I'm not a nutritionist. But I struggle with calories as the right thing to help people get to the right goal. That, you know, from my perspective and what I've seen is people have become, over the years, more resistant to that message. That we're really needing to push more that nutrient density is important.

This was further echoed by a mental health expert (\# 42):

So I think that's where the barrier is, is understanding how do we translate this idea of eating fewer calories to people, and that's why people have tried messages like, "Leave half your plate" - or "Have half your plate be greens or vegetables and fruits," so that you sort of inherently are sort of emphasizing lower-calorie food. So they're not eating less, per se, but they're eating higher quality food.

Most experts recognized that eliminating certain food might be harder than increasing healthier options. Experts believed that by adding healthier options, people would naturally reduce their consumption of less healthy food. One nutrition expert (\# 45) summarized,

Focus more on the foods that they can eat more of that we know are less calorie-dense. If they focus on incorporating more of those foods in their diet then what naturally will happen is that it will crowd out other foods that are more 
calorically dense and they'll be full from eating [these] foods and will naturally, probably over the course of a day, end up eating less.

Another obesity prevention expert (\# 4) agreed on the need to focus on what people can add to their diets:

I really try to focus on what people can add versus what they can subtract. I think that adding a healthy [behavior] tends to result in a lot more success whether it's drinking more water or it's eating an extra serving of vegetables per day. . but it's a lot easier for most people to think about what they add than what they can subtract.

Develop the right message. In general, experts advised against generic messages focusing on calorie reduction because these tended to be difficult to understand and overly broad. As 1 communications expert (\# 33) pointed out,

Our experience in a lot of the research that we've done is general messages are vague and don't really provide specific information [and therefore] are not effective.

One mental health expert (\# 10) provided insights on how a generic calorie reduction message may be left open to interpretation:

And so although I get the public health focus on keeping it simple, there's got to be more to it because of the topic, I think. Eating less for some people could mean skip meals and don't eat, right? So there's got to be more around the advice that's being given.

Another mental health expert (\# 2) provided an example of how a generic message might not resonate with certain populations, given the cultural context of food:

So I think that it would probably have less of an impact on populations where eating is more culturally based. So for example, it might be something in Latino families where food is a very big part of life and sharing food together with family and friends is important. So if it is a general "Eat less" sort of message, I think

that that might flop in particular communities of color where culturally food might play a different role than in others.

In addition, messages should be specific and action-oriented. This was summarized by 1 obesity prevention expert (\# 31),

They want action-oriented messages that are evidence-based. That they want them to be more practical than just 'eat fewer calories.' They want them to be more directive, like, 'Drink water instead of sugar-sweetened beverages.'

A mental health expert (\# 10) provided examples of what an actionoriented message might look like,

How do you educate people on what that means in their everyday life? So, "Use smaller plates." "Only eat half of what you ordered when you got out." Like strategies, and whether it's something like, "Okay, how do we eat less? We count our calories and pay attention to getting our calories down. We reduce our portions." So you come up with some kind of also catchy way of teaching people what the five top strategies are for that. "Order small instead of large." "Use smaller plates."

Experts suggested designing general key messages and then tailoring them to a specific audience. One obesity prevention expert (\# 50) suggested tailoring messages to 1 or 2 key demographics with the skills, knowledge, and resources to execute the message:

\begin{abstract}
It's important to demonstrate some success with a certain audience, and then move on. I'm not suggesting, however, that your priority audience should necessarily be the highest need audience... [Instead] they have access to healthy food. That they have access to whatever you're asking them to do. And then demonstrating success with that, and then moving on.
\end{abstract}

One communications expert (\# 9) noted the importance of culture and literacy in tailoring nutrition-related messages:

Food is very culturally based, and so the messages have to be targeted to a culture. They have to be targeted to a literacy level.

Moreover, some experts noted that fear-based messages could prompt individuals, particularly those with a family history of chronic disease, to change behavior if they had the tools and self-efficacy to do so. As 1 mental health expert (\# 47) put it,

I mean, I could see [fear-based messages] being both potentially shaming and potentially helpful... depending on where the person is and where they're at in being accepting of change. I think the fear [message] is something that they've tried to do before and that's been really difficult, and I just can't imagine it's not something that they haven't heard before.

However, most experts shared similar views and cautioned against using fear-based messages or those that focus on an individual's weight or physical appearance.

Another mental health expert (\# 5) cautioned against the use of fearbased messages:

I think that would be irrelevant in certain populations. I know there is some literature - people have looked into fear messages. . and I think they can be polarizing in that some people might just want to switch off.

Consider timing and periodicity of health behaviors. Experts advised that people may be more receptive to a calorie reduction message that encourages action on a specific day of the week. Most experts agreed that adults tend to overeat during the weekends, therefore a message targeting calorie reduction at the beginning of the week, particularly on a Monday, may be appealing. One obesity prevention expert (\# 5) best captured these views:

I wonder if it would be easier for people on, say, Monday based on the idea that maybe some 
people have overindulged a little bit at the weekend, and then they're kind of pulling themselves back together on Monday. I think it probably would be different for different people, so some people might kind of feel that effect and feel kind of good and empowered and rested after the weekend and therefore have more willpower to kind of do something like that on Monday, whereas some people might find Monday overwhelming with the beginning of the work week, and so that actually might be a bad time for them to do it. For them it might be Wednesday. So, I like the idea of maybe eat less on one day, and you pick the day based on what you think your capacity would be.

A mental health expert (\# 10) added:

But I think, just like most people kind of think of January as a kind of restart, I think that most people think of Monday as a restart.

\section{Dissemination of a Calorie Reduction Campaign}

Dissemination is an important component of any public health campaign. Experts emphasized the value of multiple dissemination channels to reach intended audiences but were split on the most appropriate spokesperson for a messaging campaign to reduce caloric intake.

Use multiple channels for dissemination. Experts identified several channels to disseminate a calorie reduction message. They suggested disseminating messages at the point of decision making (such as restaurants or hospital cafeterias) and through various channels to reach intended audiences. Experts stated that different dissemination channels would reach different audience segments and suggested mass media, such as radio, billboards, and television to reach older adults and communities of color. In addition, experts noted the popularity and reach of social media to address a wide range of audiences.
One communications expert (\# 54) recommended,

[pick] a generation to focus on. And I think there's a big difference between how we work in urban and rural communities. So, I just kind of recommend honing-in on that to help decide on settings. We certainly try and disseminate our messages across a wide variety of platforms and through schools and businesses working with work sites to get everything out there. I think the portion control specifically there's so much that we could do in the advertising space where people are looking for restaurant ads or that kind of thing. So maybe being able to advertise digitally on the apps that people are using for ordering foods or for looking up restaurants.

One communications expert (\# 9) emphasized the importance of working with the food industry to help with calorie reduction and portion control:

It's counterintuitive to them, but I actually think the restaurant industry, the food industry probably has to be engaged in this, because that would be really effective if the whole world rolled it back to a reasonable portion size and you had to buy ten of something to get a big portion.

Another communications expert (\# 1) echoed the importance of the food industry but also that of media outlets:

It's where people also eat food on an instant. So restaurants; let's see, the food industry could help out. But also TV. If you have money for TV campaigns and ads, I think that would be awesome as well.

An obesity expert (\# 6) highlighted the potential to disseminate a calorie reduction message in places where mindless eating occurs:

I mean I think it's the people and the situations where people are inadvertently eating or they're unconsciously eating, that's the population, those are the people that would benefit the most, so really targeting those situations $I$ think would be most effective.

Another obesity expert (\# 8) discussed the utility of social media and strategic partnerships to disseminate a calorie reduction message:

I think it's social media. I think Twitter, Facebook, Instagram are essential. I think a Web site that has fabulous alternative recipes that interacts with those social media sites. I think public-service announcements are effective on television still. I think partnerships. I mean, Johns Hopkins can see whether there are organizations, the ADA or other places like that, that might want to make a joint campaign with them. That would be helpful. The ADA, American Diabetes Association.

A mental health expert (\# 5) highlighted the use of social media to successfully target individuals:

I suppose that is something one could potentially do through social media, is use techniques to learn a bit more about the person and then kind of target the kind of message at that person.

Use trusted sources for dissemination of information. Experts had mixed opinions on who should deliver a calorie reduction message. Many believed that a calorie reduction message should come from a trusted professional, such as health professionals. As 1 obesity prevention expert (\# 15) said,

I think if you can get that messaging to come from someone that they're familiar with, someone that they connect with already, whether that's a physician that they trust, a nurse practitioner that they trust, someone that they're already seeing on a regular basis. I think that's when it would be most impactful when that kind of messaging, those kind of tools are coming from a place that they already have familiarity with.

Another obesity expert (\# 7) mentioned using health care providers as 
a way to efficiently reach the target audience:

[I] think you could widely disseminate it but I think you'd end up with lots of waste in terms of the audience that you're trying to reach... If you were doing a more targeted approach by trying to reach people through health care providers or through other means, that might be a better way.

The same expert (\# 7) added information on the effectiveness of patient-provider interactions on patient health behaviors:

So, one of the things that we've learned in doing our work with health care providers is when a medical professional says to a patient, "You need to do $X$ and here's a way you can do that," that that has great validity and credibility to most patients... If a doctor says to a patient or a medical professional, "You need to eat less and here's how you do that," that's something I think a lot of people will take to heart.

A mental health expert (\# 42) echoed how using health care providers as sources of the message may result in more people taking action:

\begin{abstract}
...we've seen that when we use providers, even just to get people healthcare providers - to get people to enter an intervention, that type of messaging can be more relevant to them and they're more likely to report back to their provider that they're doing these things, and if the provider is connected in, I think it can be a really helpful message. I think if you have a campaign message on a subway that said "Eat Less," I don't know if that's going to really hook people in.
\end{abstract}

Other experts thought that messages should come from peer groups, rather than experts, to reduce the potential for messages to come across as patronizing. As 1 obesity prevention expert (\# 41) explained,

I think that's part of the who's delivering the message. So, you want who's delivering the message to be people that look and sound and are our peers, as opposed to [someone] people can't relate to.

Another obesity expert (\# 40) emphasized the need for information to come from peers:

There's a lot of evidence that kind of the peer-to-peer and so if you were saying, "Here's what I do," and it was somebody that was relatable to the population that you're targeting and they're saying, "Hey, when I'm in a restaurant, I ask them to box up half of my meal," or "I save money and split a meal with my kid, my spouse," my somebody, that that could resonate...So I think peerto-peer might be one way to soften it a little bit and make it relatable and not just we're telling you what to do.

A communications expert (\# 44) highlighted the need for information to come from a trusted source whether that be a health care provider or a trusted organization:

You know,...sort of being able to connect this to other existing resources in the community, I think, is important, whether it be, you know, folks that are trying to get folks to cook healthier, maybe in a more culturally appropriate and sensitive way. I think connecting it with - I think institutions and organizations that they trust, to help them do this. Whether - in some cases it could be a health provider. Maybe it is your they're the faith-based institutions that already exist in the community. I think it's one of those, where I think - as we've learned doing this work that, you know, not only is it the type of message, but I think it's also who delivers the message, and sort of who is attached to the message.

One mental health expert (\# 2) described the need for information to come from both peer networks and trusted organizations:

Well, I think that you need, if you're going to do any sort of campaign like this, you need to have it be, as any good marketer knows, in multiple settings and delivered in multiple ways. So I think whatever sort of effective message that you would come up with, you need to make sure that it's delivered not only by, you know, an individual's network, like their family and their friends, to say, "Hey, I saw this-" wherever, or shared on social media or things like that, but you'd also want it to be communicated by organizations that they know and trust.

\section{DISCUSSION}

In this qualitative study of 60 obesity prevention, mental health, and health communications experts, researchers identified barriers and strategies to implementing an effective public health messaging campaign to reduce caloric intake in adults within the US. The barriers included the complex social and environmental factors that influence intake; lack of actionable strategies to effectively reduce calories; and the existence of too many, often conflicting, messages. Experts believed that campaign developers should consider eating behaviors and patterns, emphasize nutrient density, make the message appealing, and consider tailoring messages to their target audience, in addition to timing and periodicity in message delivery. Many experts suggested using multiple channels for dissemination and carefully considering the source of the message, and some cautioned that messages should not negatively affect vulnerable groups.

Experts identified several potential barriers to delivering a public health messaging campaign to reduce caloric intake. Understanding and accounting for the complex social and environmental influences on dietary practices were noted as the primary factors to consider when developing a calorie reduction campaign. As previous research points out, social determinants that build the social context that influences individual behavior affecting health and public health messaging campaigns are less effective if they fail to address social determinants such as race and ethnicity, socioeconomic status, social integration, and place. ${ }^{19}$ Most experts cautioned that failure to address these factors could stigmatize certain groups 
of people or lead to unintended consequences, such as disordered eating, feelings of shame, or apathy.

Obesity prevention messages tend to target the general population ${ }^{20}$ because weight gain is prevalent across the age, race, and sex spectrum. ${ }^{21}$ Nonetheless, certain groups may receive these messages differently. Previous research shows that health messaging campaigns have the potential to stigmatize overweight or obese individuals and people with eating disorders if they focus on body weight or physical appearance. $^{22,23}$ Message campaigns that focus on weight loss have the poorest weight loss outcomes. ${ }^{24}$ Fear-based messages have the potential to engage audiences with shocking and provocative images and taglines, but they may be limited in changing behavior unless people believe they have the self-efficacy to make a change. $^{25,26}$ Messages that employ scare tactics or focus on an individual's weight can shame overweight and obese persons and even imply a loss in social standing. ${ }^{23,26,27}$ Instead, people may be more receptive to messages that promote increased self-efficacy compared with messages that evoke feelings of personal blame. $^{28}$

In this study, experts believed that most people lacked general dietary knowledge, particularly as it relates to appropriate portion sizes and calorie consumption. Given this overall lack of knowledge, many people may not be as motivated to change their portion sizes or overall intake. This view is supported by studies that find that a lack of knowledge and misinformation about appropriate portion sizes are the primary barriers to behavior change. ${ }^{29,30}$ Previous studies have shown that people tend to underestimate their calorie intake and overestimate the quality of their diet. ${ }^{31,32}$ Experts thought that a lack of knowledge about healthy portion control strategies contributed to people's willingness to follow fad diets. Another study found that obese adults who were aware of healthy weight loss strategies reported more meaningful weight loss than those that followed fad diets. ${ }^{33}$

Experts noted that the current food environment is oversaturated with dietary messages that may be conflicting and lead to both confusion and apathy. This finding is consistent with other studies that have noted the influence of various groups, such as food advertisers, public health organizations, and others whose messages and competing interests can lead to mixed messages. ${ }^{15,19}$

Experts suggested several strategies to improve the success of a campaign to reduce caloric intake. First, experts suggested designing messages that addressed individual eating patterns and behaviors. Experts emphasized strategies to plan meals (such as eating on a schedule, portioning meals ahead of time) and improve dietary habits (such as eating a balanced meal and practice mindful eating) that most people could easily adopt. All experts believed that, although most people understood the link between obesity and calories, they were unsure on how to introduce simple and actionable strategies to reduce calories in their diet. In addition, some experts suggested segmenting strategies by demographics and behavior profiles to improve message receptivity, as supported by Kazbare et al. ${ }^{34}$

Second, experts thought a public health campaign to reduce calorie intake should emphasize diet quality and consumption of nutrient-rich food, rather than broadly reducing calorie intake. This finding is consistent with other studies, which showed that consuming nutrient-rich food in lieu of unhealthy options was an effective strategy to reduce overall calorie intake. $^{35,36}$ Moreover, this strategy aligns with previous research on message framing which suggested that people are more receptive to positive messages that encourage healthy behaviors and highlight the benefits of adopting certain behaviors. In a metaanalysis of 94 peer-reviewed articles evaluating the impact of message framing on attitudes, intentions, or behavior, researchers found that gainframe messages that highlighted the benefits of adopting a certain behavior were more persuasive at preventing negative health outcomes than lossframe messages or the consequences of failing to engage in a particular behavior. ${ }^{37}$ Other studies have found that loss-frame messages were only advantageous to individuals who already considered themselves to be at risk for developing a particular disease. ${ }^{37,38}$

Third, experts suggested designing appealing, simple, and visually engaging dietary messages as a way to cut through the noise in an oversaturated message environment. Welldesigned messages play a vital role in the success of a public health messaging campaign. ${ }^{39}$ Messages that are visually complex and those that offer persuasive imagery have the potential to engage with individuals with low literacy and in hard-to-reach communities. ${ }^{40,41}$

Fourth, most experts favored disseminating messages in the beginning of the week. Studies examining weekly periodicity cues have found that more people are likely to seek out or engage in healthy behaviors in the beginning of the week, particularly on a Monday. ${ }^{42,43}$ Additional research found that periodic messaging interventions have the potential for short-term behavior change and that periodic cues coupled with actionable strategies were associated with positive outcomes. ${ }^{44}$

Finally, to improve message receptivity, experts suggested disseminating messages through several platforms and through health care providers and social networks. This finding is consistent with other studies that showed that family doctors are the most frequently cited information source for nutrition and physical activity topics. ${ }^{45}$ Health professionals can be strong advocates for portion control strategies when advising patients to improve their diets. ${ }^{46}$

This study adds to the body of literature on effective messaging for calorie reduction campaigns. However, this study had some limitations. First, the research team collected only limited demographic information about experts. Therefore, researchers were not able to compare responses by geographic region or sex, for example. Second, the study sample of 60 health experts may not have represented the views of all health and nutrition experts. However, previous empirical studies have found that 9-12 interviews per population of interest is adequate to reach thematic saturation. ${ }^{47,48}$ The 
team reached thematic saturation across the 3 domains suggesting that the results from this study are generalized to similar experts in obesity prevention, mental health, and health communications. Third, the research team employed snowball sampling methods, which may have resulted in selection bias and likely decreased the diversity of the respondent pool. The research team did not differentiate between the individuals referred directly from the original sample (once-removed) and those more than once-removed from the original sample, further limiting the ability to assess the diversity of the sample. The use of snowball sampling may exclude those with smaller social networks or those with generally unpopular views. This may have biased the study results because those experts' voices and perspectives were not represented in the study. Fourth, the interview questions may have introduced bias in the study findings because the questions prompted experts to provide information on barriers and strategies to implementing a specific calorie reduction message. Fifth, the researchers did not conduct any formal checks on validity or reliability. This may have introduced bias in the findings; however, the research team worked through a process of discussion and final consensus to determine final coding scheme and themes presented. Finally, this portion of the formative research only included experts, therefore the authors cannot speak to the potential receptivity of the suggested strategies by any intended audience.

\section{IMPLICATIONS FOR RESEARCH AND PRACTICE}

Mass public health campaigns are a potentially inexpensive and effective way to encourage caloric reduction. However, very little information is provided on how formative evaluation findings are incorporated into campaign design and delivery. ${ }^{13}$ The most common formative research methods include focus groups and advisory group meetings either with the intended audience or with health experts. ${ }^{13}$ Most formative campaigns employ a small advisory committee of experts to assist in concept design. ${ }^{9,13}$ Engaging health professionals in the design, implementation, and dissemination of a public health messaging campaign can improve message receptivity, improving people's eating habits, and potentially influence food policy. ${ }^{49}$ Nonetheless, information available on formative research evaluation on nutrition campaigns is generally limited, and the results from these evaluations are not reported.

Although not purposefully grounded in theory, the findings presented in this study have theoretical implications. The results support the importance of paying attention to the components of communication theory, ${ }^{50}$ in the development of health communication campaigns. The respondents made clear the importance of developing an appropriate message, tailored to the recipient, while being mindful of contextual factors, which might interfere with message acceptance. In addition, the study findings support grounding campaigns in the social cognitive theory to encourage behavior change. ${ }^{51}$ Specifically, respondents highlighted the importance of building self-efficacy by providing individuals with the necessary knowledge to make behavior changes, while ensuring the environment and social networks allow for and encourage behavior change to happen.

Public health messaging campaigns can be informed by interdisciplinary expert feedback, which could be used to improve effectiveness. Gaining expert feedback in conjunction with message testing with the intended audience can help reduce unintended consequences and improve receptivity of the message. Engaging a variety of health experts from various content areas, including nutrition, mental health, and health communications, can help ensure that messages are widely accepted by diverse audiences. The results from this study add to the body of literature on formative research evaluation for public health nutrition campaigns. The findings can be used by public health professionals, nutrition programs, and health care providers to design effective public health messaging campaign to reduce caloric intake.

\section{REFERENCES}

1. Young LR, Nestle M. The contribution of expanding portion sizes to the US obesity epidemic. Am J Public Health. 2002;92:246-249.

2. Ledikwe JH, Blanck HM, Khan LK, et al. Low-energy-density diets are associated with high diet quality in adults in the United States. J Am Diet Assoc. 2006;106:1172-1180.

3. Zlatevska N, Dubelaar C, Holden SS. Sizing up the effect of portion size on consumption: a meta-analytic review. $J$ Mark. 2014;78:140-154.

4. McCrory MA, Harbaugh AG, Appeadu S, Roberts SB. Fast-food offerings in the United States in 1986, 1991, and 2016 show large increases in food variety, portion size, dietary energy, and selected micronutrients. I Acad Nutr Diet. 2019;119:923-933.

5. Livingstone MBE, Pourshahidi LK. Portion size and obesity. Adv Nutr. 2014;5:829-834.

6. Rolls BJ, Morris EL, Roe LS. Portion size of food affects energy intake in normal-weight and overweight men and women. Am J Clin Nutr. 2002;76: 1207-1213.

7. Herman CP, Polivy J. Normative influences on food intake. Physiol Behav. 2005;86:762-772.

8. Herman CP, Polivy J. External cues in the control of food intake in humans: the sensory-normative distinction. Physiol Behav. 2008; 94:722-728.

9. Croker H, Lucas R, Wardle J. Clusterrandomised trial to evaluate the 'Change for Life' mass media/ social marketing campaign in the UK. BMC Public Health. 2012;12:404.

10. Gase LN, Barragan NC, Robles B, Leighs M, Kuo T. A mixed-methods evaluation of the choose less, weigh less portion size health marketing campaign in Los Angeles County. Am J Health Promot. 2015;29:e214-e224.

11. Morley B, Niven P, Dixon H, et al. Population-based evaluation of the 'LiveLighter' healthy weight and lifestyle mass media campaign. Health Educ Res. 2016;31:121-135.

12. Noar SM. A 10-year retrospective of research in health mass media campaigns: where do we go from here? J Health Commun. 2006;11:21-42. 
13. Kite J, Grunseit A, Bohn-Goldbaum E, Bellew B, Carroll T, Bauman A. A systematic search and review of adult-targeted overweight and obesity prevention mass media campaigns and their evaluation: 2000-2017. J Health Commun. 2018;23:207-232.

14. Samuels and Associates for the California Obesity Prevention Program. Evaluation of San Francisco's Social Marketing Campaign "Pouring on the Pounds". Oakland, CA: Samuels and Associates; 2010. https:// www.iccp-portal.org/sites/default/files/ multimediaresources/San\%20Francisco_ Pouring_on_the_Pounds_Report.pdf. Accessed May 2, 2019.

15. Fitzgibbon M, Gans KM, Evans WD, et al. Communicating healthy eating: lessons learned and future directions. J Nutr Educ Behav. 2007;39(suppl 2):S63-S71.

16. Wakefield MA, Loken B, Hornik RC. Use of mass media campaigns to change health behaviour. Lancet. 2010;376: 1261-1271.

17. Johnson-Taylor WL, Yaroch AL, KrebsSmith SM, Rodgers AB. What can communication science tell us about promoting optimal dietary behavior. $J$ Nutr Educ Behav. 2007;39(suppl 2):S1-S4.

18. Vermeer WM, Steenhuis IH, Seidell JC. Portion size: a qualitative study of consumers' attitudes toward point-ofpurchase interventions aimed at portion size. Health Educ Res. 2010;25:109-120.

19. Viswanath K, Bond K. Social determinants and nutrition: reflections on the role of communication. J Nutr Educ Behav. 2007;39(suppl 2):S20-S24.

20. Yancey AK, Kumanyika SK, Ponce NA, et al. Population-based interventions engaging communities of color in healthy eating and active living: a review. Prev Chronic Dis. 2004;1:A09.

21. Fryar C, Kruszon-Moran D, Gu Q, Ogden C. Mean body weight, height, waist circumference, and body mass index among adults: United States, 1999-2000 through 2015-2016. Natl Health Stat Report. 2018:1-16.

22. Puhl RM, Schwartz MB, Brownell KD. Impact of perceived consensus on stereotypes about obese people: A new approach for reducing bias. Health Psychol. 2005;24:517-525.

23. Lydecker JA, Galbraith K, Ivezaj V, et al. Words will never hurt me? Preferred terms for describing obesity and binge eating. Int J Clin Pract. 2016;70: 682-690.

24. Veerman JL, Barendregt JJ, van Beeck EF, Seidell JC, Mackenbach JP.
Stemming the obesity epidemic: A tantalizing prospect. Obesity (Silver Spring). 2007;15:2365-2370.

25. Riet JvT, Ruiter RAC, Smerecnik C, Vries Hd. Examining the influence of self-efficacy on message-framing effects: reducing salt consumption in the general population. Basic Appl Soc Psych. 2010;32:165-172.

26. Fairchild AL, Bayer R, Colgrove J. Risky business: New York City's experience with fear-based public health campaigns. Health Aff (Millwood). 2015; 34:844-851.

27. Ellis S, Rosenblum K, Miller A, Peterson KE, Lumeng JC. Meaning of the terms "overweight" and "obese" among low-income women. J Nutr Educ Behav. 2014;46:299-303.

28. Puhl R, Luedicke J, Lee PJ. Public reactions to obesity-related health campaigns: A randomized controlled trial. Am J Prev Med. 2013;45:36-48.

29. Steenhuis IH, Vermeer WM. Portion size: review and framework for interventions. Int $J$ Behav Nutr Phys Act. 2009;6:58.

30. Young LR, Nestle M. Reducing portion sizes to prevent obesity: a call to action. Am J Prev Med. 2012;43:565-568.

31. Variyam JN, Shim Y, Blaylock J. Consumer misperceptions of diet quality. $J$ Nutr Educ. 2001;33:314-321.

32. Sorensen MR, Matthiessen J, Holm L, Knudsen VK, Andersen EW, Tetens I. Optimistic and pessimistic self-assessment of own diets is associated with age, self-rated health and weight status in Danish adults. Appetite. 2017;114:15-22.

33. Nicklas JM, Huskey KW, Davis RB, Wee CC. Successful weight loss among obese U.S. Adults. Am J Prev Med. 2012;42:481-485.

34. Kazbare L, van Trijp HCM, Eskildsen JK. A-priori and post-hoc segmentation in the design of healthy eating campaigns. J Mark Commun. 2010;16:21-45.

35. Troesch B, Biesalski HK, Bos R, et al. Increased intake of foods with high nutrient density can help to break the intergenerational cycle of malnutrition and obesity. Nutrients. 2015;7:60166037.

36. Gardner CD, Trepanowski JF, Del Gobbo LC, et al. Effect of low-fat vs low-carbohydrate diet on 12-month weight loss in overweight adults and the association with genotype pattern or insulin secretion: the DIETFITS randomized clinical trial low-fat vs low-carbohydrate diet on weight loss in overweight adults low-fat vs low-carbohydrate diet on weight loss in overweight adults. JAMA. 2018;319:667-679.

37. Gallagher KM, Updegraff JA. Health message framing effects on attitudes, intentions, and behavior: A meta-analytic review. Ann Behav Med. 2011;43:101116.

38. Maheswaran D, Meyers-Levy J. The influence of message framing and issue involvement. J Mark Res. 1990;27:361367.

39. Wilson BJ. Designing media messages about health and nutrition: what strategies are most effective? J Nutr Educ Behav. 2007;39(suppl 2):S13-S19.

40. Niederdeppe J, Bu QL, Borah P, Kindig DA, Robert SA. Message design strategies to raise public awareness of social determinants of health and population health disparities. Milbank Q. 2008;86: 481-513

41. Lazard AJ, Mackert MS. E-health first impressions and visual evaluations: key design principles for attention and appeal. Commun Q Rev. 2015;3: 25-34.

42. Ayers JW, Althouse BM, Johnson M, Cohen JE. Circaseptan (weekly) rhythms in smoking cessation considerations. JAMA Intern Med. 2014;174: 146-148.

43. Welding K, De Leon E, Cha S, Johnson M, Cohen JE, Graham AL. Weekly enrollment and usage patterns in an Internet smoking cessation intervention. Internet Interv. 2017;9:100-105.

44. De Leon E, Fuentes LW, Cohen JE. Characterizing periodic messaging interventions across health behaviors and media: systematic review. J Med Internet Res. 2014;16:e93.

45. van Dillen SM, Hiddink GJ, Koelen MA, de Graaf C, van Woerkum CM. Understanding nutrition communication between health professionals and consumers: development of a model for nutrition awareness based on qualitative consumer research. Am J Clin Nutr. 2003;77(suppl 4):1065S1072S.

46. Institute of Medicine, Food and Nutrition Board, Committee on Accelerating Progress in Obesity Prevention. Accelerating Progress in Obesity Prevention: Solving the Weight of the Nation. Washington, DC: National Academies Press; 2012.

47. Namey E, Guest G, McKenna K, Chen M. Evaluating bang for the buck: a cost-effectiveness comparison between 
individual interviews and focus groups based on thematic saturation levels. $A m$ J Eval. 2016;37:425-440.

48. Guest G, Bunce A, Johnson L. How many interviews are enough?: an experiment with data saturation and variability. Field Methods. 2006;18: 59-82.
49. Miller GD, Cohen NL, Fulgoni VL, Heymsfield SB, Wellman NS. From nutrition scientist to nutrition communicator: why you should take the leap. Am J Clin Nutr. 2006;83:1272-1275.

50. Shannon CE, Weaver W. The Mathematical Theory of Communication. Champaign, IL: University of Illinois Press; 1963.
51. McAlister AL, Perry CL, Parcel GS. How individuals, environments, and health behaviors interact: social cognitive theory. In: Glanz K, Rimer BK, Lewis FM, eds. Health Behavior and Health Education: Theory, Research, and Practice. 3rd ed. San Francisco, CA: Jossey-Bass; 2002:169-188.

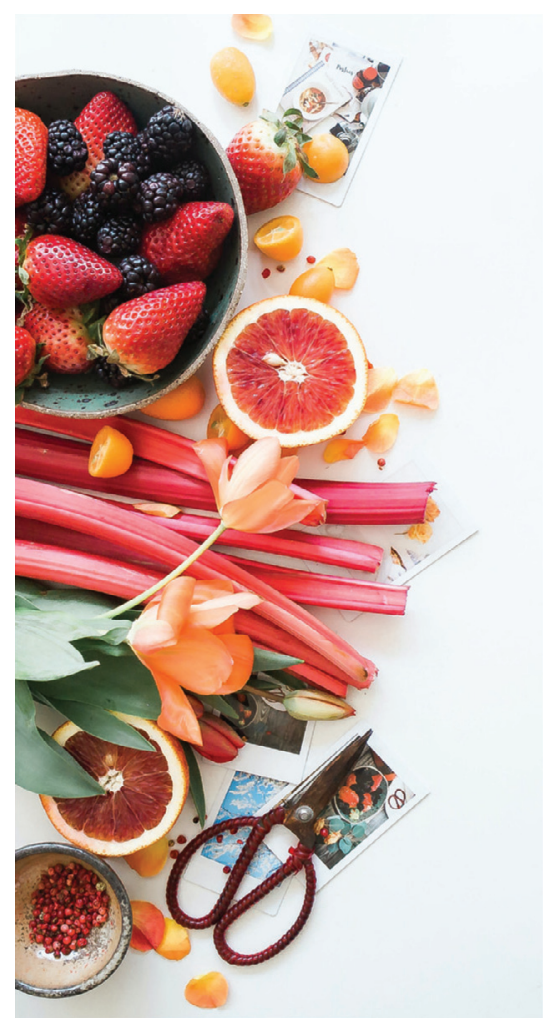

\section{Submit GEMs to JNEB}

Great Educational Materials (GEMs) are brief descriptions of innovative and useful approaches to nutrition education and behavior. GEMs include a description of the teaching technique, activity, or material; implementation procedures; and evidence of usefulness or impact. Submit your next GEM to JNEB.

Questions? Review detailed author instructions online or contact managingeditor@jneb.org.

www.jneb.org 